\title{
A re-examination of the Stratton effect: Egocentric adaptation to a rotated visual image ${ }^{1}$
}

\author{
ARIEN MACK, ${ }^{2}$ GRADUATE FACULTY, NEW SCHOOL FOR SOCIAL RESEARCH \\ IRVIN ROCK, INSTITUTE FOR COGNITIVE STUDIES, RUTGERS UNIVERSITY
}

The question raised by Stratton, whether a disoriented image can yield upright vision, was explored by exposing $O s$ to a prismatically rotated field. To isolate the problem of adaptation to altered egocentric orientation, the $O$ 's view was restricted to a horizontal plane during both prism exposure and test. Significant adaptation based on movement-derived information was obtained.

In 1896 George Stratton published a report on a series of experiments designed to determine whether the inverted retinal image is necessary for normal upright perception (Stratton, 1896). Despite these now classic experiments and a string of frequentlycited other experiments, the answer to this question is not yet clear. We suggest the reason for this is not only the intrinsic difficulty in performing the appropriate experiment, but that the exact nature of the problem has never been clearly understood.

The question of whether or not a particular orientation of the retinal image is necessary for normal perception is a question about the relationship of image orientation to the perception of directions relative to the self (egocentric direction). Therefore an experiment designed to answer that question must be one in which not only (1) image orientation is changed through rotation of the retinal image, but (2) exposure to the rotated retinal image must occur under conditions free from variables that might contaminate the effect, and (3) measurements of the adaptation must be exclusively sensitive to changes in the perception of egocentric direction.

To elucidate points 2 and 3: The problem raised by Stratton is primarily concerned with the perception of egocentric directions and should not be confused with the question of the perception of directions relative to the main axes of space (environmental direction). Terms like "up" and "down," "vertical" and "tilted" are ambiguous in that they can be thought of in relation to the $O$ or in relation to the spatial environment. Ordinarily, with the $O$ upright, the sets of directions are congruent; what is upward in relation to the $\mathrm{O}$ is also upward in the environment (e.g., the sky); what is vertical in the environment is also parallel to the sagittal plane of the O's body (egocentric vertical). But when the $O$ is tilted or in a plane parallel to the ground (e.g., supine or bending over looking down at the ground), these sets of directions are quite separate and distinct. If, for example, the $O$ is tilted, then a line parallel to his body (egocentric vertical) is clearly not one which is vertical in the environment; if he is supine, what is up and down in the egocentric sense is quite distinct from what is up and down in the environment (Rock, 1954).

We believe the question Stratton posed is properly concerned with egocentric direction since the optical system that disorients the retinal image is attached to the O's head. Therefore, regardless of his position in space, the optical device consistently alters directions in relation to himself. If the $O$ views the world through lenses or prisms while he remains upright, then a blurring or confusion of the theoretical picture is likely. To illustrate: if a tilting (rather than an inverting) device is worn, then the scene will at first appeared tilted. That is, the scene will appear tilted in relation to the observer. Suppose that after a period of exposure the $O$ reports that the world appears less tilted and measurements taken by means of a tilting line which the $O$ set to "vertical" confirms this. What does "less tilted" mean? It might well mean less tilted from the vertical of space. Indeed Wertheimer (1912) long ago showed that such an effect-a tilted scene appearing less tilted than it actually is-occurs more or less immediately. Conceivably over time an effect such as this might increase. Yet this would not necessarily indicate anything about how the orientation of things had changed in relation to the self, or, if it did, it might indicate a change based upon changes in perceived environmental direction. For this reason, it would not be a desirable procedure for isolating the problem raised by Stratton.

We turn now to the question of the basis of adaptation to a disoriented image. Stratton wrote, "Some visual objects may be inverted with respect to other visual objects, but the whole system of visual objects can never by itself be either inverted or upright" [Stratton, 1897, p. 476]. The point being made here is that whenever the entire image is rotated all the relationships within the image are maintained. The image of the feet is still closest to the ground and that of the head closest to the sky. Inversion entails a relationship between two or more objects, one of which is inverted with respect to the other. With respect to what visual object, then, may we say that the entire image is inverted?

The logic of this reasoning leads to the prediction that when an $O$ looks through an optical device which rotates the entire retinal image, he should at once perceive the world normally. Things should continue to appear right side up, yet as everyone knows who has ever looked through such an optical system, the world appears either upside down or tilted. Some explanation is required of the fact that the world looks disoriented at the very beginning of experiments utilizing such optical systems.

Failing to see how the inversion could be within the visual system itself when the internal relationships in that system are unchanged, Stratton was apparently forced to conclude that the perceived inversion was between vision and some other sense modality. We maintain, however, that it is both a simpler and more accurate description of the facts to assume that the inversion occurs within the visual system itself. We suggest that the inversion is to be accounted for by the fact that a particular retinal direction has come to signify a particular egocentric direction and that this relationship is altered by image rotation. For example, the successive stimulation of lower to upper retina normally signifies that an object is moving egocentrically down in the direction of forehead to chin to feet. Now on looking through an inverting lens system from a normal upright position, an object actually moving in this direction will move across the retina in exactly the opposite direction. It will therefore signify to the $O$ that the object is moving egocentrically upward. The crucial assumption is that the nervous system retains a record of absolute image orientation, the stimulus-copy aspect of the trace, bound via associations to a particular egocentric direction (Rock, 1966). Note that this association refers to egocentric and not environmental directions. Through the naked eye a line which casts its image in the retinal vertical direction will always be parallel to the long axis of the head (egocentrically vertical), but that same line will not necessarily be environmentally vertical. That is why an association can be formed between retinal and egocentric direction but cannot be formed between retinal and environmental direction. Adaptation occurs when there has been an appropriate shift in the significance of retinal-cortical directions.

In order to study the Stratton problem it is, of course, not necessary to rotate the image $180 \mathrm{deg}$. Since the question is whether the normal orientation of the image is necessary for upright perception, any consistent change in the orientation of the image should allow us to investigate the problem. Most of the work on this problem, including Stratton's own, involved a $180 \mathrm{deg}$ image rotation. Actually this seems ill-advised, since the likelihood of achieving any adaptation in a short period of time would appear to be enhanced by lessening the severity of image rotation. Furthermore, where the image is rotated less than $180 \mathrm{deg}$, gradual adaptation seems possible in a way which does not appear possible when there has been complete inversion. 
The few experiments that have. been reported involving adaptation to a tilted retirial image make no reference to the Stratton problem and unfortunately none of them provides unambiguous answers to it (Mikaelian \& Held, 1964; Morant \& Beller, 1965; Ebenholtz, 1966). In these experiments the measure of adaptation involved perception of the vertical of environmental space, and, the exposure to the rotated image occurred under the typical condition where the $O$ walked around in a normal environment. Thus the measured perceptual changes could have been instances of the Wertheimer effect (Wertheimer, 1912) entailing adaptation to the altered directions within environmental space, or the change could reflect a normalization to tilted lines such as was studied by Gibson (1937). In either case the changes could not be considered unambiguous evidence of egocentric change. 3

\section{EXPERIMENT 1}

The purpose of this experiment was to determine whether or not the normally oriented retinal image is necessary for upright perception, defined egocentrically. This experiment was repeated many times over a three year period and each repetition or minor variation always involved a fresh subject population.

\section{Method}

The conditions of exposure ruled out the possibility of any adaptation to the altered directions of environmental space, and were such that they permitted study of egocentric adaptation to a rotated image. The prismatic rotation of the image in no way affected the coordinates of environmental space. This was accomplished by restricting the S's view during prism exposure to a horizontal plane. Throughout exposure the $S$ was required to walk with his head bent over, parallel to the floor, and to follow a circuitious path indicated by tape glued to the floors of the laboratory corridors. The $S$ was constantly monitored to be certain that his head was never raised while his eyes were open and he was wearing prisms. Under these conditions the prism rotation could in no way influence the main environmental coordinates, since the tilt was orthogonal to the direction of gravity. Any adaptation that occurs must entail a change in egocentric orientation only.

Exposure lasted $1 / 2 \mathrm{~h}$ divided into two $15 \mathrm{~min}$ periods which were separated by a few minutes of rest in a completely dark room Throughout the entire experiment the S's left eye was occluded and during exposure he wore a pair of right angle prisms in tandem set in a goggle frame over his right eye (Mack, 1966). Half the Ss were exposed to a 25 deg clockwise and half to a counterclockwise rotation. Prior to and immediately following prism exposure the $S$ was required to make four judgments of egocentric orientation. The difference between these pre- and post-judgments provided the measure of adaptation.

The measure of egocentric orientation was obtained in a manner consistent with the exposure conditions. Again the S's head was bent over parallel to the floor, but now it was held in one position by a bite plate covered with dental impression wax. His task was, while in complete darkness, to set a luminous dot placed directly below his head and slightly above the floor to the "twelve o'clock" position. He was able to do this by turning a knob placed in his right hand. Prior to each judgment the $\mathrm{E}$ placed the dot in a starting position which was either $\mathrm{cw}$ or $\mathrm{ccw}$ from $120^{\prime} \mathrm{clock}$. This order was counter-balanced. The apparatus consisted of a circular piece of white poster board which was placed parallel to the floor and was mounted on a small wooden frame. At the center of the circle was a luminous dot. A second luminous dot was placed toward the periphery of the circle 3 in. from the center. The disc could be rotated about a vertical axis by a knob which was connected to it by a simple gear system. When rotated in the dark, the outer dot appeared to move around the stationary center dot describing the circumference of an undelineated circle in the way that the hands of a clock rotate about the center of the clock face. The position of the outer dot was read from a degree scale inscribed on the edge of the posterboard which, of course, was not visible to the $S$ in the dark. A bite plate attached to a rigid horizontal bar was located directly above the center dot, and served to minimize the possibility of slight shifts in head position both within a testing session and from one testing session to the next. It was extremely important to keep the head in one position throughout testing since even a slight change in head position could effect the perceived location of the 12 o'clock position. This is so because the egocentric vertical with which the 12 o'clock position is more or less synonymous is determined with reference to the head.

The task of setting a point to 12 o'clock under these conditions can be considered a reliable index of egocentric orientation because no position in a horizontal plane in which the dot moves is distinguishable from any other with respect to sensed differences in gravitational pressure. All points in a horizontal plane are orthogonal to gravity and, therefore, the 12 o'clock position must be determined solely by the egocentric significance of a particular retinal direction. Twelve $o^{\prime}$ clock must under these conditions be that position in which the two dots appear to be aligned with the saggital plane of the head. We chose this 12 o'clock task rather than one in which the $S$ was explicitly instructed to set the points so that they were aligned with the long axis of his head, because in some trial runs with these instructions, the $S$ was uncertain about the location of this position whereas he seemed immediately certain where 12 o'clock was.

If adaptation to a rotated image occurs, we would expect a shift in the perception of 12 o'clock in the direction of the prism's rotation, since adaptation means that a new retinal direction now signifies the egocentric vertical. Complete adaptation to an image rotated $25 \mathrm{deg} \mathrm{cw}$ would be indicated by a setting of the point to 12 o'clock after prism exposure that was $25 \mathrm{deg} \mathrm{cw}$ of the pre-exposure setting and partial adaptation would be indicated by any setting that differed from the pre-exposure setting in a $\mathrm{cw}$ direction.

Since this procedure was a novel one, it was necessary to be certain that Ss were able to perform the required task with a reasonable degree of consistency. To this end we performed a preliminary experiment in which the measuring procedure was identical to that in the main experiment but no prisms were used in the exposure phase. The two sets of judgments, analogous to the pre- and post-judgments of the main experiment, were separated by an interval of $1 / 2 \mathrm{~h}$ in which the $S$ was free to walk about as he pleased. Eight Ss performed the task. The mean change without regard to direction between the earlier and later judgments for all Ss was $2.5 \mathrm{deg}$, a change which is not significant $(t=2.0)$. The differences both within and between Ss' judgments were sufficiently small to establish that the technique was adequate.

\section{Subjects}

Twenty Ss performed the main experiment. This included both male and female students between the ages of 18 and 30 from colleges in New York City. All Ss were unfamiliar with the purpose of the experiment and were paid for their participation.

\section{Results}

The mean aftereffect for all $20 \mathrm{Ss}$ was $4.7 \mathrm{deg}$, which is significant at the .01 level. Table 1 presents the mean aftereffects for each $S$ and the combined means for $\mathrm{cw}$ and $\mathrm{ccw}$ prism rotation. The results reveal a shift in egocentric orientation following exposure to a rotated image, and thus provide ananswer to Stratton's problem. The normally oriented retinal image does not appear to be necessary for veridical perception of egocentric direction. Further corroboration of these results comes from two other experiments that resemble this one in every essential and which were performed during the course of our investigation of this problem. One of the experiments involved $16 \mathrm{Ss}$ and yielded a mean aftereffect of $6.28 \mathrm{deg}$. The other experiment involved only five Ss and yielded a mean aftereffect of $5.71 \mathrm{deg}$. Of the $31 \mathrm{Ss}$ in the three experiments all except one showed a pre- to postexposure shift in the judgment of 12 o'clock in the predicted direction. 
Table 1

The Mean Difference Between Pre and Post Prism Exposure Judgments of 12 o'clock

CW Prism Exposure**

\begin{tabular}{|c|c|c|c|}
\hline Subjects & Mean Difference* & Subjects & Mean Difference* \\
\hline 1 & -4.50 & 11 & 6.00 \\
\hline 2 & $-5,00$ & 12 & 8.49 \\
\hline 3 & -2.00 & 13 & 2.00 \\
\hline 4 & -3.75 & 14 & 1.00 \\
\hline 5 & -7.50 & 15 & 1.12 \\
\hline 6 & -5.75 & 16 & 3.00 \\
\hline 7 & -6.37 & 17 & 3.50 \\
\hline 8 & -2.00 & 18 & 12.00 \\
\hline 9 & -3.50 & 19 & 9.62 \\
\hline 10 & 4.75 & 20 & 2.25 \\
\hline
\end{tabular}

*All differences are in degrees.

** A minus difference predicted.

***A plus difference predicted.

\section{Discussion}

While the results of this experiment do seem clearly to indicate the presence of egocentric adaptation, there is one argument that counters this interpretation. It might be claimed that the effect obtained is the result of a process of normalization to an atypical posture. This interpretation of adaptation aftereffects was suggested by Hein (1965) in an attempt to deal with apparent evidence of adaptation obtained with stationary exposure to a laterally displaced image. Applied to the conditions of exposure present in this experiment, the argument would have to be that while wearing the prisms the $S$ feels compelled to twist his head in order to align it with his visible feet which in this instance appear to be rotated $25 \mathrm{deg}$ from the egocentric vertical. Since we made no effort to restrain left or right head turning, only the down position of the head having been monitored, some attempt to deal with the possible consequences of such an atypical posture must be made. The argument continues-if the head is turned to the left or right, depending on the direction of the prism, then the turned head position might in a short time come to feel normal, i.e., unturned, with the result that when the S's head is no longer in this position and is back in its usual unturned position, as it is during testing, he may now feel that his head is turned in the opposite direction. Were this to happen, the $S$ might now place the luminous dot in a direction opposite to that of his presently-felt head position, which would be $\mathrm{cw}$ for $\mathrm{Ss}$ exposed to $\mathrm{cw}$ prism rotation and $\mathrm{ccw}$ for those exposed to $\mathrm{ccw}$ rotation. Such setting would then provide spurious evidence of egocentric adaptation. If, however, Ss were to do this, it must mean that the judgment of 12 o'clock is made with reference to the sagittal plane of the torso and not the head, something we assumed not to be the case.

A variant of this argument which also might account for these results is one proposed by Harris and commonly referred to as a "proprioceptive" theory of adaptation (Harris, 1965). According to this argument all adaptation is the result of a recalibration of proprioception. The prism induced visual distortion produces a conflict between vision and touch in which vision dominates and remains unchanged, effecting a change in felt position of a part or parts of the body which is in line with their new visual location. Harris had not yet explicitly tried to explain adaptation to a rotated image in these terms, but the interpretation of these results along the lines of proprioceptive theory might be as follows. As the result of viewing through prisms which rotate the visual image, the S's legs, fect, etc., appear turned with respect to his shoulders or head. This apparent turn is at odds with the proprioceptive input. The result of the visual-proprioceptive conflict is that the $S$ may come to experience the normal position of his head as turned in a direction opposite to the apparent position of his feet and legs. There will then be a recalibration of proprioception from the head or, more properly, the neck, such that only that position of the head which is aligned with the apparent rotation of the feet and legs will feel straight ahead of the shoulders. From this point the argument is identical with that of "atypical posture." The $\mathrm{S}$ who has been exposed to $\mathrm{cw}$ rotation when placed in the testing position where his head is actually straight ahead of his shoulders will set the luminous point in a cw direction to compensate for the felt $\mathrm{ccw}$ turn of his head. Again this argument assumes that the perception of 12 o'clock is determined by the sagittal plane of the trunk and not by the head alone.

In order to test these explanations of the results of Experiment 1 , a second experiment was performed to determine the effects of an atypical relation between head and torso on the perception of the egocentric vertical. If either of these interpretations are to have any bearing on the results, it is necessary to establish that the perception of the egocentrically determined 12 o'clock is governed by the sagittal plane of the trunk and not by the head alone.

\section{EXPERIMENT 2}

\section{Method}

Since the only purpose of this experiment was to ascertain the effect, if any, of an altered head-trunk relationship on the perception of the egocentric vertical, no prism exposure was used. Immediately after the pre-test judgments were completed (the procedure for testing was identical to that of the previous experiment), the $S$ was instructed to close both his eyes and to turn his feet in either an extreme cw or ccw direction while his head position was maintained by the bite plate. This maneuver had the desired effect of drastically altering the relationship between head and torso. If feeling one's head to be turned when it is actually straight effects the perception of 12 o'clock, then actually turning the head ought to have a similar or even stronger effect. In this new posture the $S$ proceeded to make four new judgments of the 12 o'clock positon.

\section{Subjects}

There were eight Ss, both male and female who were students at one of the universities of New York City. Half of the Ss rotated their feet $\mathrm{cw}$ and half $\mathrm{ccw}$.

\section{Results}

Table 2 presents the results. Of the four $\mathrm{Ss}$ in the cw group two shifted in the wrong direction, one showed no change, and one shifted in the predicted direction by $3.5 \mathrm{deg}$. Of the four Ss in the ccw group, two showed minor shifts in the wrong or non-predicted direction and one showed a large shift in that direction. The one $\mathrm{S}$ in this group whose setting shifted in the predicted direction did so by a mere $.13 \mathrm{deg}$. The overall mean shift is not significant.

\section{DISCUSSION}

The results indicate that a change in the normal relationship between head and torso does not cffect the perception of the egocentric vertical. Judgments of the 12 o'clock position of a luminous point in the dark appear to be based on its perceived

Table 2

Control for Atypical Posture and/or Proprioceptive Change

\begin{tabular}{ccc} 
Ss & Mean Difference & Rotation of Feet \\
\hline 1 & $-2.5^{\circ} *$ & $\mathrm{cw}$ \\
2 & $3.5^{\circ}$ & $\mathrm{cw}$ \\
3 & $0.0^{\circ}$ & $\mathrm{cw}$ \\
4 & $-2.0^{\circ}$ & $\mathrm{cw}$ \\
5 & $-2.0^{\circ}$ & $\mathrm{ccw}$ \\
6 & $-3.75^{\circ}$ & $\mathrm{ccw}$ \\
7 & $-11.15^{\circ}$ & $\mathrm{ccw}$ \\
8 & $.13^{\circ}$ & $\mathrm{ccw}$ \\
\hline Mean & -2.23 & \\
\hline
\end{tabular}

*A minus indicates a difference opposite to that predicted. 
orientation with respect to the head. The orientation of the head with respect to the trunk appears to be irrelevant. It would, therefore, seem that neither an atypical posture nor a proprioceptive account of the results of Experiment 1 are relevant. It therefore seems reasonable to conclude that these results are evidence of a shift in the retinal correlate of the egocentric vertical. A new retinal orientation has come to signify that direction which appears parallel to the head.

Once having established the adaptation effect, we began investigating the factors which are operative in producing it. Is the sensory information derived from movement necessary for adaptation to occur? There is reason to believe it is at least important if not necessary. In an experiment previously reported (Mack, 1966), it was found that movement information is important for adaptation to a rotated visual image under conditions which, however, were not analytic about whether the adaptation obtained was to the vertical of space or the egocentric vertical. The experimental work done by Held and his colleagues supports this view.

In order to avoid a theoretical confusion it is necessary to distinguish between the movement information which is in principle at least, available whenever there is movement, and reafferent stimulation which is the consequence of active movement and which Held has argued is essential to the adaptation process. In the experiment cited above, movement information was found to be important to adaptation; reafferent stimulation was not.

Movement can provide information about the egocentric orientation of objects in the visual field derived from the transformation of the retinal image correlated with movement in a given direction. An example may make this clear. In Experiment 1, as the $S$ begins to follow the taped paths, he will almost always make an error in the direction of the prism rotation. If the path actually stretches straight in front of him and he is wearing cw rotating prisms, he will err by moving off to his right in the apparent direction of the path. Before very long he will, of course, find himself off the path. Only if he moves straight ahead will he be able to successfully follow it. During this bit of maneuvaring he has gained the information that an image of an object which strikes the retina on a cw oblique is, in fact, parallel to the long axis of his head, for only if he walks straight forward where the path apparently veers to his right will he manage to stay on it. Our view is that this kind of information can result in a shift in the retinal correlate of the egocentric vertical necessary for egocentric adaptation.

The next experiment was concerned with determining whether or not movement information is crucial to egocentric adaptation. Because the many experiments performed almost always yielded either ambiguous or negative results, they will be described in less than full detail and the several variations of a given experiment will be described together (this will be made clear in each instance).

In principle we believe egocentric adaptation to a rotated image can occur in the absence of movement. This should be possible if the information derived from movement is made available in some other way, since it is our conviction, supported at least in part by experimental evidence, that it is the information derived from movement and not the movement per se which is crucial (Mack, 1966; Rock, 1965, Howard, Craske, \& Templeton, 1965; Weinstein, Sersen, Fisher, \& Weisinger, 1964). In the case of adaptation to a rotated visual image the $S$ must obtain information that a new retinal direction indicates the egocentric vertical. Since the egocentric vertical is defined as that direction parallel to the sagittal plane of the head and/or body, then permitting the $S$ sight of his head and body while wearing prisms ought to provide him with direct information of the kind necessary for adaptation. The fact that the image of the head when, for example, the head is seen in a mirror, now falls on a retinal oblique rather than on the retinal vertical would seem to be the most immediately relevant kind of information possible. The following experiment was based on this reasoning. It involved stationary Ss who looked at themselves either directly or through a mirror during prism exposure. In all the variations of the experiment the egocentric character of the predicted adaptation was guaranteed by keeping the S's head parallel to the floor throughout all phases of the experiment.

\section{EXPERIMENT 3}

\section{Method}

The measurement procedure was the same as that of Experiment 1. During the exposure period which in most of the experiments was $15 \mathrm{~min}$, the $S$ continued to bite on the same bite plate used during the test. This made certain that the head remained in the same position from the beginning to the end of the experiment. The S stood with his head down and with one eye looked at himself through a single right angle prism set slightly in front of the bite apparatus. ${ }^{4}$ The other eye was covered by a patch. In all the experiments there was a rather large mirror on the floor immediately in front of the S's feet. This enabled the S to see his head and upper torso reflected in the mirror. He was able to see his lower torso and feet directly. The degree of prism rotation was changed in the several variations of this experiment. It was either 30,50 or $60 \mathrm{deg} \mathrm{cw}$ or $\mathrm{ccw}$. In the initial experiments, all the $S$ was required to do during the exposure phase was to look at himself. However, standing in one place, with head down while biting on dental wax is both uncomfortable and tedious; therefore in some of the later experiments a task was introduced in an attempt to encourage the $S$ to continue to focus his attention. In one variation of this task the $S$ was asked to indicate when a letter printed on a circular white disc placed directly in his line of sight looked to be in the best position for reading. In another variation the $S$ was asked to indicate when a line drawn on a circular piece of cardboard appeared either parallel or perpendicular to his head. The $S$ was never informed about whether such settings were right or wrong. Neither of these tasks had any noticeable effects. In the most successful variant of this experiment the prisms were used throughout, so that all testing was done with the prisms. This technique was tried in an effort to shorten the interval between exposure and test and to insure that there was no change of head position from pre-to post-test. Despite the wax impression of the teeth, the $S$ might not have placed his head in precisely the same position in the post-test as it had been in the pre-test. When the prism was removed after the exposure period, it was necessary for the $S$ to lift his head from the bite plate and to place it back on the bite plate only after the prism was removed.

\section{Subjects}

A total of 59 Ss were tested in the several variations. Of these, 20 performed in the experiment in which the prisms remained in place throughout. All Ss were students in colleges in and around New York City and were paid for participating.

\section{Results}

The main conclusion to be drawn from these results is that sight of the self under these experimental conditions is not generally effective in producing a shift in the perception of the egocentric ver tical. This appears to be so despite the fact that the experiment in which the prisms were used throughout yielded a mean aftereffe:t of $4.7 \mathrm{deg}$ and nine out of $10 \mathrm{Ss}$ who were exposed to a $\mathrm{cW}$ and seven out of 10 exposed to a ccw prism rotation showed positive adaptive shifts. The prism rotation in this particular variation was $50 \mathrm{deg}$. Had the results in the other experiments resembled these, the conclusion would, of course, have been quite different. It is because these results appear to be an exception rather than the rule, and because testing with prisms is a less stringent method, that we conclude that adaptation under stationary conditions does not reliably occur. However, an interesting sidelight of these experiments was the fact that many Ss immediately accepted the tilted view of themselves as normal, that is, many Ss were unaware of the prism rotation. This appears to be further evidence of immediate correction to prismatic distortion and can be thought of as immediate adaptation (cf., Rock, Goldberg, \& Mack, 1966). If so, the problem arises of why 
there is no reliable aftereffect. The single most perplexing aspect of this experiment is the frequency with which the prism exposure led to a particular direction of change in the perception of 12 o'clock. Frequently, regardless of the direction of prism exposure, the pre- to post-shift was $\mathrm{cw}$. Thus when the exposure happened to be $\mathrm{cw}$, the results appeared positive, but when the exposure was $\mathrm{ccw}$ the results were negative. We have been unable to account for this phenomenon, but are at least certain that it is a reliable one since we encountered it repeatedly while testing under these conditions.

\section{Discussion}

It is only possible to speculate about why no consistent evidence of an appropriate egocentric adaptation was obtained. These results by and large do not support our interpretation of the basis of adaptation. It now appears that only sensory information which is available under dynamic conditions is consistently effective. Regardless of how logically important a particular kind of information seems to be, in this case sight of the self, it is not always effective when it is presented statically. If, however, it is legitimate to generalize from the results of experiments on adaptation to other kinds of visual distortion, such a conclusion is questionable (Wallach, Kravitz, \& Lindauer, 1963; Rock, Goldberg, \& Mack, 1966; Rock, 1965; Howard, Craske, \& Templeton, 1965). In any case, these results cannot be viewed as evidence for the necessity of active movement, for only an experiment which established the superior effectiveness of active over passive movement lends support to that hypothesis.

An effort was made to determine whether active movement was more conducive to adaptation than passive movement but the method it was necessary to employ resulted in no adaptation in either the active or passive condition. It is possible that the difference between this experiment and the earlier ones lies in the reduced size of the visual field for the $S$ was confined to a cart and was able to see only a small area of the floor at any moment while in the initial experiment there was no such restriction. If this is true, it is not clear why decreasing the size of the visual field should result in the disappearance of the aftereffect.

\section{EXPERIMENT 4}

In our analysis of how movement can provide information about the prismatic tilting of the image, no reference was made to the $S$ seeing parts of his own body. The underlying assumption was that the direction of flow of the retinal image provided the crucial information. Yet in our experiment, at least in the ccw condition, the S typically does see his feet and part of his legs because the prism system also produces a slight displacement in one direction. Does this play a crucial role? Despite the more or less negative results of Experiment 2, it is entirely possible that sight of the body in interaction with movement derived information would prove effective. To explore the question more rigorously, the following procedure was employed.

\section{Method}

The $\mathrm{S}$ was required to wear a semi-circular collar around his waist. It projected outward 8 in. from his waist with the result that in looking downward at the floor it was impossible to see any part of his feet or legs. The collar itself was seen, but its circular shape afforded no clues as to which part of it was adjacent to which part of the body. It did not, however, materially restrict the view of the floor. To make comparisons with previous experiments more meaningful, the ccw position of the prism was used. This was the condition in which, in previous experiments, the Ss could see their feet. In all other respects the procedure was identical to that of Experiment 1.

\section{Subjects}

Ten Ss were used, recruited from colleges in New York City. They were all paid for participation.

\section{Results}

The mean difference between pre- and post-judgments of 12 o'clock for the 10 Ss was $4.12 \mathrm{deg}(t=5.15$ which is significant beyond the .001 level).

\section{Discussion}

The effect obtained was quite comparable to that of previous experiments in which the feet were visible. We conclude that sight of the feet neither helps nor hinders adaptation to an egocentrically tilted field. This means that the information in Experiment 1 is based exclusively on movement. Replications of the procedure entailing wearing of the collar failed to reveal any consistent effect of this factor even when the same S served under both "collar" and "no-collar" conditions.

\section{CONCLUSION}

From the results of Experiment 1 it is clear that Stratton was correct. The inverted retinal image is not necessary for normal upright perception. The perception of the egocentric vertical shifted consistently after exposure to a visual image rotated $25 \mathrm{deg}$. Although the recorded shift was only partial, representing a compromise between the old and new egocentric meaning of retinal direction, the assumption is made that extending the exposure period beyond $1 / 2 \mathrm{~h}$ would, in time lead to complete adaptation, so that only an object whose image struck the retina at a 25 deg angle would appear egocentrically vertical. This, however, is an assumption and demands experimental proof for its acceptance.

Having established the existence of pure egocentric adaptation, we went on to show that information based on movement seemed to be both necessary and sufficient to achieve this effect. However, changes of the conditions of exposure, such as restricting the size of the field or increasing the amount of tilt resulted in washing out the effect. We are therefore left with unanswered questions. That sight of the self while wearing prisms is not conducive to adaptation runs counter to our theoretical convictions. We are also left without an explanation for the cw bias repeatedly obtained during stationary exposure.

This series of experiments thus produced a definitive answer to a long unanswered question and turned up a battery of other unanswered questions.

\section{REFERENCES}

EBENHOLTZ, S. Adaptation to a rotated visual field as a function of optical tilt and exposure time.J. exp. Psychol, 1966, 72, 629-634.

HARRIS, C. Perceptual adaptation to inverted, reversed and displaced vision. Psychol. Rev., 1965, 72, 419-444.

HEIN, A. Postural aftereffects and visual motor adaptation to prisms. Paper read at 1965 MIT conference on adaptation.

HOWARD, I. P., CRASKE, B., \& TEMPLETON, W. B. Visumotor adaptation to discordant exafferent stimulation. J. exp. Psychol, 1965, 70, 189-191.

MACK, A. The role of movement in perceptual adaptation to a tilted retinal image. Percept. \& Psychophys., 1967, 2, 65-68.

MIKAELIAN, H., \& HELD, R. Two types of adaptation to an optically rotated field. Amer. J. Psychol, 1964, 257-263.

MORANT, R., \& BELLER, H. K. Adaptation to prismatically rotated visual field. Science, $1965,148,530-531$.

ROCK, I. The perception of the egocentric orientation of a line. J. exp. Psychol, 1954, 69, 513-528.

ROCK, 1. The noture of perceptual adaptation. New York: Basic Books, 1966.

ROCK, I. Adaptation to a minified image. Psychon. Sci, 1965, 2, 105-106.

ROCK, I., GOLDBERG, J., \& MACK, A. Immediate correction and adaptation based on viewing a prismatically displaced scene. Percept. \& Psychophys, 1966, 1, 351-354.

ROELOFS, C. O. Optische lokalisation. Arch. Augenheilk, 1935, 109, 395-415.

STRATTON, G. M. Some preliminary experiments on vision without inversion of the retinal image. Psychol Rev., 1896, 3, 611-617.

STRATTON, G. M. Vision without inversion of the retinal image. Psychol Rev., 1897, 4, 341-360, 463-481.

WEINSTEIN, S., SERSEN, E. A., FISHER, L., \& WEISINGER, M. Is reference necessary for visual adaptation? Percept. mot. Skills, 1964, 18. $641-648$. 
WERTHEIMER, M. Experimentelle studien uber das sehen von bewegung. $Z$. Psychol, 1912, 61, 161-265.

\section{NOTES}

1. We gratefully acknowledge the help of Nili Hillman, Joel Goldberg, Diane Iandoli and Anita Kushner who assisted in the running of the experiments reported.

2. Address, Department of Psychology, Graduate Faculty, New School for Social Research, 66 West 12th Street, New York, N. Y. 10011.

3. Gibson and others since have demonstrated that a tilted line will appear less tilted following a period of inspection and a vertical line will subsequently appear tilted in the opposite direction. Since a normal scene viewed through prism yields many images of vertical lines in the environment that are tilted on the retina, the conditions for a Gibson normalization effect would seem to be present in prism adaptation experiments, and therefore an attempt must be made to either eliminate such an effect or allow for it. In the experiment by Mikaelian and Held (1964) the exposure to prisms which rotated the image occurred while the $\mathbf{O}$ was in a dark room which contained no visible straight lines, but only randomly placed luminous spheres. The adaptation effect they obtained could therefore not be attributed to normalization. Further proof was provided by a test in which the $S$ was required to adjust two luminous points, one slightly above and the other slightly beiow eye level, to the apparent straight ahead. Normalization would not be expected to affect the perceived direction of a point.

4. We were concerned with the left-right reversal produced by the single prism because the Ss were unable to detect the reversal when looking down at their stationary, symmetrical bodies.

(Accepted for publication March 21, 1968.) 